\title{
Financial earthquakes, aftershocks and scaling in emerging stock markets
}

\author{
Faruk Selçuk* \\ Department of Economics, Bilkent University, Bilkent, Ankara 06800, Turkey
}

Received 14 October 2003

\begin{abstract}
This paper provides evidence for scaling laws in emerging stock markets. Estimated parameters using different definitions of volatility show that the empirical scaling law in every stock market is a power law. This power law holds from 2 to 240 business days (almost 1 year). The scaling parameter in these economies changes after a change in the definition of volatility. This finding indicates that the stock returns may have a multifractal nature.

Another scaling property of stock returns is examined by relating the time after a main shock to the number of aftershocks per unit time. The empirical findings show that after a major fall in the stock returns, the stock market volatility above a certain threshold shows a power law decay, described by Omori's law.
\end{abstract}

(c) 2003 Elsevier B.V. All rights reserved.

PACS: $\mathrm{C} 1 ; \mathrm{G} 0 ; \mathrm{G} 1 ; \mathrm{F} 3$

Keywords: Volatility; Scaling; Stock market; Emerging markets; Omori’s law; Multifractality

\section{Introduction}

For some years, the equilibrium models of financial markets, such as the Capital Asset Pricing Model [1,2] and the Arbitrage Pricing Theory [3], and the efficient market hypothesis have dominated the finance literature [4,5]. A common feature of empirical work in this area is that the analysis is conducted keeping the time interval fixed. Also, market participants are usually regarded as homogeneous entities with identical time horizons.

\footnotetext{
* Tel.: +90-532-294-8796; fax: +1-208-694-3196.

E-mail address: faruk@bilkent.edu.tr (F. Selçuk).
} 
In the early 1960s, Mandelbrot [6] introduced fractal models to describe certain features of financial and economic time series. In this approach, the dynamics of a given time series is analyzed at different time scales (daily, weekly, monthly, etc.). In other words, the object (time series) is viewed at different resolutions and certain characteristics are noted at each resolution. As a result, certain regularities have been discovered in most of the economic and financial data leading to further research to explain these regularities. Recent studies on multiscale analysis of financial and economic time series using new techniques [7-10] provide further empirical support to the idea that the agents have different time horizons and that they operate at different time scales. In other words, homogeneous market participants operating at a single time scale is a rare exception, rather than a rule, according to empirical work.

Multiscale analysis of economic and financial data points out certain "scaling laws", such as a "pure power law" [11-18]. Although some of the empirical scaling laws do not say anything specific about the data generating stochastic process, they are useful because (i) they stimulate the search for interpretive frameworks, (ii) they impose discipline on theory formation (the theory must generate data consistent with the observed scaling result), (iii) (they) give clues to properties of the space of possible underlying data generating process [19].

Recently, some studies claim that visual power laws and empirical long memory reported in the finance literature might be an artifact. LeBaron [20] provided a simple stochastic volatility model which is able to produce visual power laws and long memory similar to those from actual return series using comparable sample sizes. He pointed out that these are small-sample features for the stochastic volatility model, since asymptotically the model does not posses these properties. However, Stanley and Plerou [21] showed that the simple stochastic volatility model provided by LeBaron [20] cannot produce power laws and long memory. (See, also, other articles on this issue in the November 2001 issue of Quantitative Finance.)

Scaling behavior of financial time series is investigated by employing different methods in different studies [7,11-17,22-24]. One approach is to estimate the tail index. The tail index estimation is accomplished by keeping the time interval of returns constant and investigating the behavior of the tails of the distribution. For example, Gençay and Selçuk [8] studies the tail behavior of the return distributions in emerging markets (same sample used in this study) in a value-at-risk framework. According to tail index estimations in Ref. [8], the return distributions in emerging markets are fat-tailed and the fat-tailness of returns is much bigger than what is observed in developed markets.

Another approach to study the scaling behavior of financial time series is accomplished by examining the dynamics of volatility (defined as absolute returns) as a function of the time interval on which the time series is measured. Matteo et al. $[15,16]$ use this approach to estimate the Hurst exponent of different financial time series in several developed and developing economies. They show that the deviations from pure Brownian motion is associated with the degree of development of the markets. Particularly, they found that there is a clear tendency for mature liquid markets to have a Hurst exponent less than or equal to 0.5 , whereas less developed markets shows a tendency to have a Hurst exponent significantly greater than 0.5 [15]. 
Recently, a series of papers investigates the behavior of volatility in financial markets before and after big crashes. An early work by Sornette et al. [25] showed that the implied volatility in the S\&P500 after the 1987 financial crash has a power-law-periodic decay. More recently, Lillo and Mantegna [26,27] showed that number of S\&P500 index returns above a large threshold following a large shock is well described by a power law function which is analogous to Omori's law in geophysics.

In this study, the scaling behavior of emerging stock market returns is investigated by examining the dynamics of volatility (defined as absolute returns) as a function of the time interval on which the returns are measured. Furthermore, the scaling behavior of volatility is examined for different powers of the absolute return series. This enables us to obtain some information about the multiscaling and changing distribution characteristic of the emerging stock market returns. Another contribution of the paper is that we discover a scaling law of volatility after a major shock. Specifically, it is shown that the volatility (described as absolute daily returns) above a certain threshold shows a power-law decay after a major crash in these markets. The next section introduces the data set and reports certain scaling properties of this sample. We conclude afterwards.

\section{Data and estimation}

\subsection{Preliminary analysis}

We study daily stock market data from Argentina, Brazil, Hong Kong, Indonesia, Korea, Mexico, Philippines, Singapore, Taiwan and Turkey. The sampling period ends in December 2000 for each country. The beginning date is determined according to data availability in datastream, and extends as far back as 1973 (Hong Kong and Taiwan). The tail behavior of the stock market returns in these countries has been studied previously [28]. This study provides further insight about the scaling behavior of the stock market returns in these emerging markets from a different perspective. The descriptive statistics of daily stock returns in each country are presented in Table 1 . The daily returns are defined as

$$
r_{i, t}=\log \left(x_{i, t} / x_{i, t-1}\right)=\left(\log x_{i, t}-\log x_{i, t-1}\right),
$$

where $x_{i, t}$ is the daily closing value of the stock market index in country $i$ on day $t$.

The average daily returns in Table 1 imply unusual (annual compound) returns as high as 161 percent in Brazil and 77 percent in Turkey. This result should not be surprising since these two countries experienced high rates of price inflation during the sample period. The annual rate of inflation (consumer prices) in Brazil was over 2000 percent per year in 1993 and 1994 before it was stabilized under 10 percent later. The annual rate of inflation (consumer prices) in Turkey fluctuated between 60 and 120 percent during 1988-1999.

According to the sample kurtosis estimates and the sample skewness, the daily rate of returns are far from being normally distributed. The sample kurtosis estimates 
Table 1

Descriptive statistics of the daily returns, $\log \left(x_{t} / x_{t-1}\right) \times 100$, from nine emerging stock markets

\begin{tabular}{|c|c|c|c|c|c|c|c|}
\hline & $n$ & Mean & Std & $\mathrm{Ku}$ & Sk & Min & Max \\
\hline Argentina & 1935 & 0.01 & 1.9 & 9.19 & 0.00 & -13.6 & 11.9 \\
\hline Brazil & 2086 & 0.37 & 3.0 & 10.56 & 0.54 & -17.2 & 28.8 \\
\hline Hong Kong & 7305 & 0.04 & 2.0 & 36.64 & -1.43 & -40.5 & 17.2 \\
\hline Indonesia & 2085 & 0.04 & 1.1 & 16.92 & -1.29 & -11.8 & 6.7 \\
\hline Korea & 2868 & -0.02 & 2.1 & 7.36 & 0.27 & -12.5 & 14.6 \\
\hline Mexico & 1453 & 0.06 & 2.0 & 10.90 & -0.20 & -15.0 & 13.3 \\
\hline Philippines & 1076 & -0.02 & 1.5 & 6.78 & 0.10 & -7.9 & 9.1 \\
\hline Singapore & 3910 & 0.04 & 1.4 & 61.25 & -2.21 & -29.2 & 15.5 \\
\hline Taiwan & 7305 & 0.04 & 1.9 & 11.82 & 0.09 & -19.7 & 19.9 \\
\hline Turkey & 3223 & 0.22 & 3.2 & 8.04 & 0.15 & -19.8 & 30.5 \\
\hline
\end{tabular}

$n=$ sample size; Mean = sample mean; Std=standard deviation; Ku=kurtosis; Sk=skewness; Min $=$ minimum observed daily return; Max = maximum observed daily return. Source: Datastream. Sample periods: Argentina: August 2, 1993, to December 29, 2000; Brazil: January 1, 1993, to December 29, 2000; Hong Kong: January 1, 1973, to December 29, 2000; Indonesia: January 4, 1993, to December 29, 2000; Korea: January 3, 1990, to December 29, 2000; Mexico: June 6, 1995, to December 29, 2000; Singapore: January 4, 1985, to December 29, 2000; Taiwan: January 1, 1973, to December 29, 2000; Turkey: January 8, 1988, to December 29, 2000. Data source: Datastream.

(the lowest: 6.8 in Philippines and the highest: 61.2 in Singapore) indicate that the return distributions in all the markets are fat-tailed. The sample skewness shows that the daily returns have a symmetric distribution only in Argentina. In all other countries, the returns have either positive or negative skewness. The sample skewnesses are negative in Hong Kong, Indonesia, Mexico and Singapore. This indicates that the asymmetric tail extends more toward negative values than positive ones. Positive skewness in other countries ranges from 0.09 (Taiwan) to 0.54 (Brazil).

Table 1 also shows the highest and lowest one-day return from each country. The highest one-day positive returns are in Turkey (30.5 percent) and Brazil (28.8 percent). The highest one-day losses are in Hong Kong (40.5 percent), Singapore (29.2 percent) and Turkey (19.8 percent). Notice that given the sample standard deviation and the mean of logarithmic returns, a normal distribution assumption would imply that the probability of observing a $5 \sigma$ loss in a given day (as in Korea) is less than $10^{-6}$ while the probability of observing a $10 \sigma$ loss in a given day (as in Indonesia) is $10^{-23}$. In addition to evidence provided by previous research $[15,16,28])$, the sample statistics indicate once again that the stock market returns in these countries are far from being normally distributed. Therefore, a Gaussian scaling of volatility, so called "square root" formula, is not valid and the scaling behavior of the return distributions in each market should be examined in more detail.

\subsection{Scaling and multifractality}

Following Mandelbrot [12] and Dacorogna et al. [7], the average volatility in each stock market is measured as a certain power of the absolute returns observed over 


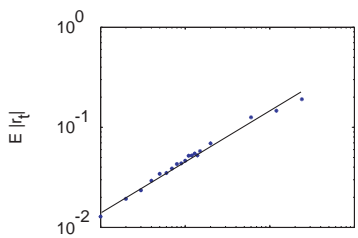

(a)

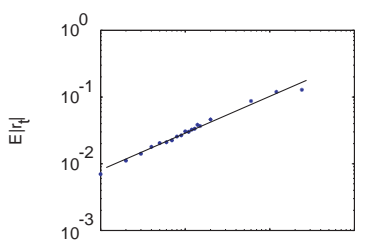

(d)

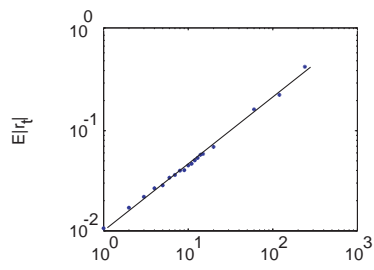

(g) Log (time interval in days)

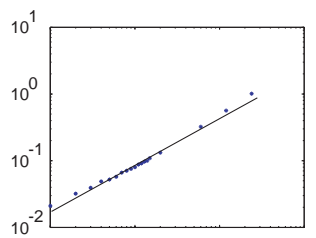

(b)

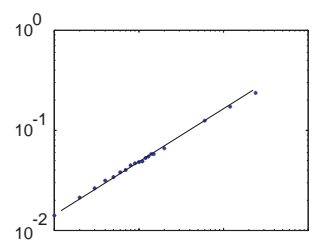

(e)

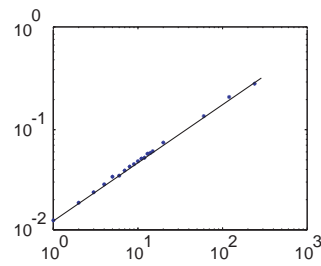

(h)

Log (time interval in days)

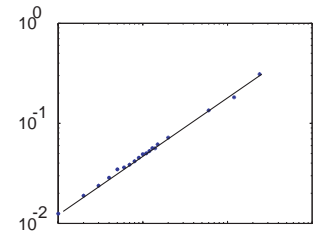

(c)

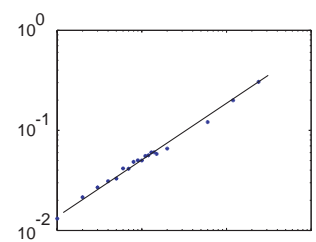

(f)

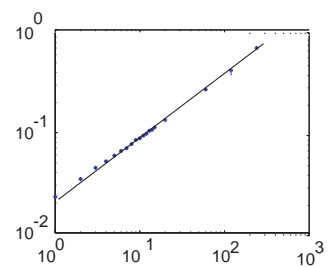

(i) Log (time interval in days)

Fig. 1. (a-i) Scaling laws in different emerging stock markets. The mean absolute return, $E\left|r_{t}\right|$, is plotted as a function of the aggregation period, $\Delta t$, on logarithmic scale in nine different emerging stock markets. The aggregation periods are $1-15,20,60,120$ and 240 business days. The last aggregation period corresponds to approximately 1 year.

different time intervals $\Delta t$ :

$$
\left\{E\left[|r|^{q}\right]\right\}^{1 / q}=c(q) \Delta t^{D(q)},
$$

where $E$ is expectations operator, $c(q)$ and $D(q)$ are deterministic functions of $q$. Dacorogna et al. [7] calls $D(q)$ the drift exponent. For a Gaussian random walk, the drift exponent is constant $(0.5)$ regardless of the choice of volatility definition (different $q \mathrm{~s})$. If the drift exponent is constant but different than 0.5 for different definitions of volatility, the process is said to be unifractal (or uniscaling). A typical indication of multifractality and changing distributions is to observe different drift exponents for different definitions of volatility.

Fig. 1 plots the $\log$ of the mean absolute return, $E\left|r_{t}\right|$, as a function of the $\log$ of the aggregation period, $\Delta t$, in nine different emerging stock markets. The aggregation periods are $1-15,20,60,120$ and 240 business days. The last aggregation period corresponds to approximately one year since there are around 260 business days in 1 year. It is clear that the empirical scaling law in every stock market is a power law 
Table 2

The estimated drift exponent $D(q)$ in Eq. (2) and 95 percent bootstrap confidence intervals

\begin{tabular}{lllll}
\hline & $D(1)$ & $D(2)$ & $D(3)$ & $D(4)$ \\
\hline Argentina & $0.55 \pm 0.03$ & $0.50 \pm 0.03$ & $0.45 \pm 0.03$ & $0.40 \pm 0.03$ \\
Brazil & $0.59 \pm 0.03$ & $0.54 \pm 0.03$ & $0.49 \pm 0.02$ & $0.43 \pm 0.03$ \\
Hong Kong & $0.57 \pm 0.01$ & $0.50 \pm 0.01$ & $0.41 \pm 0.02$ & $0.32 \pm 0.03$ \\
Indonesia & $0.56 \pm 0.03$ & $0.50 \pm 0.02$ & $0.43 \pm 0.04$ & $0.36 \pm 0.04$ \\
Korea & $0.55 \pm 0.03$ & $0.50 \pm 0.02$ & $0.45 \pm 0.03$ & $0.42 \pm 0.03$ \\
Mexico & $0.55 \pm 0.04$ & $0.50 \pm 0.04$ & $0.44 \pm 0.04$ & $0.39 \pm 0.05$ \\
Philippines & $0.55 \pm 0.04$ & $0.50 \pm 0.04$ & $0.45 \pm 0.04$ & $0.41 \pm 0.05$ \\
Singapore & $0.57 \pm 0.02$ & $0.50 \pm 0.02$ & $0.39 \pm 0.03$ & $0.29 \pm 0.04$ \\
Taiwan & $0.56 \pm 0.02$ & $0.50 \pm 0.02$ & $0.47 \pm 0.03$ & $0.43 \pm 0.03$ \\
Turkey & $0.55 \pm 0.02$ & $0.50 \pm 0.02$ & $0.47 \pm 0.03$ & $0.43 \pm 0.03$ \\
Simulation & $0.50 \pm 0.02$ & $0.50 \pm 0.03$ & $0.50 \pm 0.03$ & $0.50 \pm 0.03$ \\
\hline
\end{tabular}

The empirical drift exponent $D(1)$ in column one is significantly greater than 0.5 for all countries. This is in line with the results reported by Matteo et al. [15,16]. The empirical drift exponent changes for all countries after a change in the definition of volatility $(q=2,3,4)$. This result implies that the distributions are also changing. For sample periods and data source, see Table 1. The last row reports estimated drift exponents from a simulated random walk with sample size 3000 .

as all data points lie on a straight line. This power law holds from 2 to 240 business days (almost 1 year). ${ }^{1}$

The estimated drift exponent $D(q)$ for different values of $q$ in Eq. (2) is presented in Table 2. In order to obtain robust estimates, we changed the highest aggregation factor from 5 to $15,20,60,120$ and 240 business days in our estimations and calculated the average drift exponent. In addition, 95 percent confidence intervals and drift exponent estimates are obtained through the bootstrapping method [31]. The bootstrap is implemented by sampling without replacement from the data to produce a new data set each time and from this new data new estimates are calculated. The procedure is replicated 100 times. Reported drift exponents in Table 2 are averages of these bootstrap estimates which also utilized the changing highest aggregation period. In order to check the robustness of this procedure, we generated a random walk series with a sample size of 3000. Estimated draft exponents using the same procedure for this simulated series in Table 2, last raw show that the procedure performs well.

The empirical drift exponent $D(1)$ in column one is significantly greater than 0.5 for all countries. This is in line with the results reported by Matteo et al. $[15,16]$. When we change the definition of volatility from absolute returns $(q=1)$ to the powers of absolute returns $(q=2,3,4)$, the empirical drift exponent changes for all countries and becomes smaller with increasing $q$. Note that these volatility measures put more emphasis on the tails than simple absolute returns. Similar result have been reported earlier for foreign exchange rates of developed countries and Eurofutures in [7,17] which use the same

\footnotetext{
${ }^{1}$ Although it is not clearly visible in these plots, a small deviation from linearity is observed in some countries especially at 5 and 10 days aggregation periods. This is probably due to strong weekly seasonality in absolute returns in those countries. Although it is difficult to define an optimum seasonal adjustment procedure in general [29], a seasonal filter, as in [22,30] might be employed to extract seasonality because seasonal components are nonscaling, and may induce some bias in estimations.
} 
definitions of volatility in Eq. (2). The changing drift exponent might be interpreted as an indication of the changing form of distribution under aggregation and also as a sign of multifractality.

\subsection{Aftershocks and Omori's law}

A major earthquake in a region is usually followed by smaller ones, labelled as "aftershocks". There are several approaches to describe the dynamics of aftershocks. A well-known simple rule is the Gutenberg-Richter relation [32], which says that the number of earthquakes of magnitude $M$ or greater, $N(M)$ is given by

$$
\log N(M)=a-b M
$$

where $a$ and $b$ are two constants. In several studies, $b$ is found to be within the range of 0.7-1 regionally. However, for larger geographical areas and the world, the slope parameter $b$ is usually 1 . Notice that fitting the tails of a return distribution in finance literature is analogous to this relation.

Another approach relates the time after the main shock to the number of aftershocks per unit time, $n(t)$. This is known as the Omori law [26,27,33]. Omori's law states that the number of aftershocks per unit time decays with power law $n(t) \propto t^{-p}$. In order to avoid divergence at $t=0$, Omori's law is rewritten as

$$
n(t)=K(t+\tau)^{-p}
$$

where $K$ and $\tau$ are constants. By integrating Eq. (4) between 0 and $t$, the cumulative number of aftershocks between the main shock and the time $t$ can be expressed as

$$
N(t)=K\left[(t+\tau)^{1-p}-\tau^{1-p}\right] /(1-p)
$$

when $p \neq 1$ and $N(t)=K \ln (t / 1+\tau)$ for $p=1$ [26].

Recently, a series of papers investigates the behavior of volatility in financial markets after big crashes. An early work by Sornette et al. [25] shows that the implied volatility in the S\&P500 after the 1987 financial crash has a power law-periodic decay. More recently, Lillo and Mantegna [26,27] showed that number of S\&P500 index returns above a large threshold after a large shock in the market is well described by a power law function which is analogous to Omori's law in Eq. (5).

In our sample countries, the two largest one-day drops (in percentage terms) in the stock market during the sample period are considered to be "financial earthquakes". As a result, we have twenty major shocks from different markets. Table 3 gives the dates of these "financial earthquakes", corresponding daily percent loss, sample standard deviation and relative shocks (percent loss divided by the sample standard deviation). Each country in the sample except Hong Kong and Taiwan experienced a major shock during 1997 or 1998. The largest one day fall in percentage terms is observed in Hong Kong (33.3 percent, October 1987). The smallest one-day fall is Philippines (7.2 percent, January 1998). The relative shock measure, daily percent loss divided by the sample standard deviation, indicate that these major shocks lie within the range of $4.5 \sigma$ 
Table 3

Stock market crashes and Omori's law

\begin{tabular}{|c|c|c|c|c|c|}
\hline & Date & $\%$ Loss & $\sigma$ & Rel. Shock & $p$ \\
\hline Argentina & 27-Oct-97 & 12.7 & 1.9 & 6.7 & 1.05 \\
\hline Argentina & 10-Sep-98 & 10.3 & & 5.4 & 0.52 \\
\hline Brazil & 20-Oct-97 & 15.0 & 3.0 & 5 & 1.32 \\
\hline Brazil & 10-Sep-98 & 15.8 & & 5.3 & 1.12 \\
\hline Hong Kong & $26-O c t-87$ & 33.3 & 2.0 & 16.7 & 1.04 \\
\hline Hong Kong & 05-Jun-89 & 21.7 & & 10.9 & 0.60 \\
\hline Indonesia & 28-Oct-97 & 11.2 & 1.1 & 10.2 & 0.55 \\
\hline Indonesia & 17-Apr-00 & 7.4 & & 6.7 & 0.76 \\
\hline Korea & 24-Nov-97 & 10.6 & 2.1 & 5 & 0.89 \\
\hline Korea & 17-Apr-00 & 11.7 & & 5.6 & 0.76 \\
\hline Mexico & $02-O c t-95$ & 12.4 & 2.0 & 6.2 & 1.41 \\
\hline Mexico & 27-Oct-97 & 14.0 & & 7 & 0.65 \\
\hline Philippines & 28-Aug-97 & 7.6 & 1.5 & 5.1 & 0.58 \\
\hline Philippines & 09-Jan-98 & 7.2 & & 4.8 & 0.69 \\
\hline Singapore & $10-$ Oct- 87 & 13.1 & 1.4 & 9.3 & 0.63 \\
\hline Singapore & 28-Oct-97 & 9.2 & & 6.6 & 0.62 \\
\hline Taiwan & 14-Aug-73 & 12.5 & 1.9 & 6.6 & 0.64 \\
\hline Taiwan & 28-Mar-83 & 11.1 & & 5.8 & 1.05 \\
\hline Turkey & 03-Jan-90 & 17.9 & 3.2 & 5.6 & 0.98 \\
\hline Turkey & 11-Nov-98 & 14.5 & & 4.5 & 0.69 \\
\hline
\end{tabular}

The table shows the two largest one day fall (in percentage) in each country during the sample period. Daily percent loss and the sample standard deviation $(\sigma)$ of logarithmic returns are also shown for each country. Notice that given the sample standard deviation and the mean of logarithmic returns, a normal distribution assumption would imply that the probability of observing a $5 \sigma$ loss in a given day (as in Korea) is less than $10^{-6}$ while the probability of observing a $10 \sigma$ loss (as in Indonesia) is $10^{-23}$. The last column shows the estimated values of parameter $p$ in Eq. (5). For sample periods and data source, see Table 1. For estimation purposes, the cumulative number of aftershocks for 100 days after the main shock is employed except Indonesia (1997), Brazil (1998), Hong Kong (1989), Philippines (1998), Turkey (1990, 1998) where the estimation periods were 200 days. An aftershock is defined as a daily absolute return greater than $1 \sigma$ (the sample standard deviation).

(Turkey, November 1998) and 16.7 $\sigma$ (Hong Kong, October 1987). The dispersion of relative shocks is small since most of them are around $6 \sigma$. Assuming a standard normal distribution for the return series, the probability of observing a $6 \sigma$ loss in any given day is approximately $10^{-10}$ (assuming 250 business days in one year, it corresponds to one day in 40 million years)!

Having determined the dates of two major shocks in each country, an aftershock is defined as daily absolute return greater than $1 \sigma$ immediately after the major shock. The number of cumulative aftershocks $N(t)$ for $t=100$ is calculated and an estimate of $p$ in Eq. (5) is obtained for each country. The estimated exponent $p$ differs from country to country and from one aftershock to another aftershock period in the same country. The $p$ value is found to be within the range of 0.52 (Argentina, after September 1998 crash) to 1.41 (Mexico, after October 1997 crash). However, majority of estimated exponents (14 cases out of 20) is less than 1 , concentrating between 0.50 and 0.70 . Fig. 2 plots a sample of cumulative number of aftershocks, $N$, (dotted line) and the 


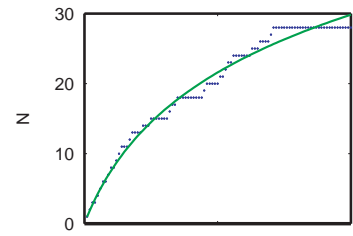

(a)

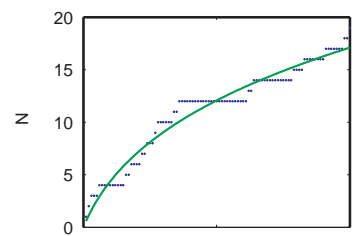

(d)

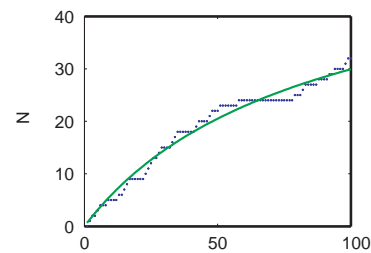

(g)

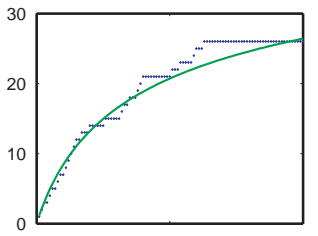

(b)

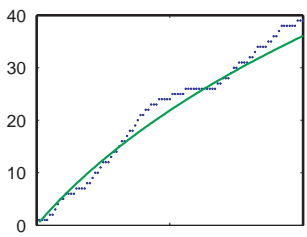

(e)

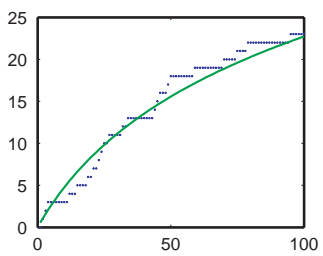

(h)

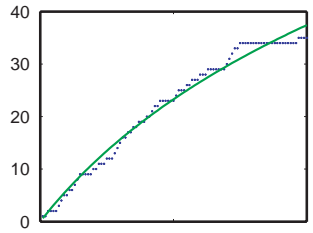

(c)

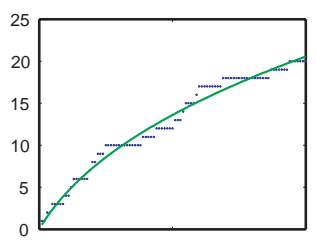

(f)

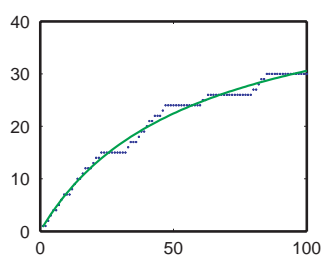

(i)

Days

Fig. 2. Cumulative number of aftershocks and Omori's Law in different emerging markets. Plots show the cumulative number of aftershocks, $N$ (dotted line) and the best fit of Eq. (5) for each market. An aftershock is defined as a realized daily absolute return greater than $1 \sigma$ (the sample standard deviation of logarithmic returns). Plots shows 100 days after the crash of (a) 27-October-1997 in Argentina, (b) 20-October-1997 in Brazil, (c) 26-October-1987 in Hong Kong, (d) 17-April-2000 in Indonesia, (e) 17-April-2000 in Korea, (f) 27-October-1997 in Mexico, (g) 9-January-1998 in Philippines, (h) 28-March-1983 in Taiwan and (i) 3-January-1990 in Turkey. These crashes are one of the two biggest daily losses in each country during the sample period. (See Table 2.)

best fit of Eq. (5) for each market. Clearly, the aftershock sequence in each market is well described by the power law function in Eq. (5).

\section{Conclusion}

This paper provides evidence for empirical scaling laws in emerging stock market returns. Estimated drift exponents for different definitions of volatility show that the empirical scaling law in every stock market is a power law. This power law holds from 2 to 240 business days (almost one year). The changing drift exponent after a change in the definition of volatility in these economies indicate that the stock returns may have a multifractal nature.

Another scaling property of stock returns in these economies is examined by relating the time after a main shock to the number of aftershocks per unit time. The empirical 
findings show that after a major fall in the stock returns, the stock market volatility above a certain threshold shows a power law decay, described by Omori's law.

\section{References}

[1] J. Lintner, The valuation of risk assets and the selection of risky investments in stock portfolio and capital budgets, Rev. Econ. Stat. (47) (1965) 13-37.

[2] W.F. Sharpe, Capital asset prices: a theory of market equilibrium under conditions of risk, J. Financ. (19) (1964) 425-442.

[3] S. Ross, The arbitrage theory of capital asset pricing, J. Econ. Theory (13) (1976) 341-360.

[4] J.Y. Campbell, A.W. Lo, A.C. MacKinlay, The Econometrics of Financial Markets, Princeton University Press, Princeton, NJ, 1997.

[5] E. Fama, Efficient capital markets: a review of theory and empirical work, J. Financ. (25) (1970) $383-417$.

[6] B.B. Mandelbrot, New methods in statistical economics, J. Political Econ. (71) (1963a) 421-440.

[7] M. Dacorogna, R. Gençay, U. Muller, R. Olsen, O. Pictet, An Introduction to High-Frequency Finance, Academic Press, San Diego, 2001.

[8] R. Gençay, F. Selçuk, B. Whitcher, An Introduction to Wavelets and Other Filtering Methods in Finance and Economics, Academic Press, San Diego, 2001b.

[9] J.B. Ramsey, The contribution of wavelets to the analysis of economic and financial data, Philos. Trans. R. Soc. London A (357) (1999) 2593-2606.

[10] J.B. Ramsey, Wavelets in economics and finance: past and future, Stud. Nonlinear Dyn. Econ. (3) (2002) 1.

[11] L. Calvet, A. Fisher, Multifractality in asset returns: theory and evidence, Rev. Econ. Stat. (84) (2002) 381-406.

[12] B.B. Mandelbrot, Fractals and Scaling in Finance, Springer, Berlin, 1997.

[13] R.M. Mantegna, H.E. Stanley, Scaling behaviour in the dynamics of an economic index, Nature (376) (1995) 563-567.

[14] R.N. Mantegna, H.E. Stanley, An Introduction to Econophysics, Cambridge University Press, Cambridge, MA, 2000.

[15] T.D. Matteo, T. Aste, M.M. Dacorogna, Scaling behaviors in differently developed markets, Physica A (324) (2003a) 183-188.

[16] T.D. Matteo, T. Aste, M.M. Dacorogna, Using the scaling analysis to characterize financial markets, J. Empirical Financ. (2003b) forthcoming.

[17] U. Muller, M. Dacorogna, O. Pictet, M. Schwarz, C. Morgenegg, Statistical study of foreign exchange rates, empirical evidence of a price change scaling law, and intraday analysis, J. Bank. Financ. (14) (1990) 1189-1208.

[18] Z. Xu, R. Gençay, Scaling, self-similarity and multifractality in FX markets, Physica A (323) (2003) $578-590$.

[19] W.A. Brock, Scaling in economics: a reader's guide, Ind. Corporate Change (8) (1999) 409-446.

[20] B. LeBaron, Stochastic volatility as a simple generator of apparent financial power laws and long memory, Quant. Financ. (1) (2001) 621-631.

[21] H.E. Stanley, V. Plerou, Scaling and universality in economics: empirical results and theoretical interpretation, Quant. Financ. (1) (2001) 563-567.

[22] A. Fisher, L. Calvet, B. Mandelbrot, Multifractality of Deutschemark-US dollar exchange rates, Cowles Foundation Discussion Paper No. 1165, Yale University, 1997.

[23] B. Mandelbrot, A. Fisher, L. Calvet, A multifractal model of asset returns, Cowles Foundation Discussion Paper No. 1164, Yale University, 1997.

[24] B.B. Mandelbrot, The variation of certain speculative prices, J. Bus. (36) (1963b) 394-419.

[25] D. Sornette, A. Johansen, J.P. Bouchaud, Stock market crashes, precursors and replicas, J. Phy. I (6) (1996) $167-175$.

[26] F. Lillo, R.N. Mantegna, Dynamics of financial market index after a crash, Manuscript, (2002) http://arXiv.org/abs/cond-mat/0209685. 
[27] F. Lillo, R.N. Mantegna, Power law relaxation in a complex system: Omori law after a financial market crash, Phys. Rev. E 68 (2003) 016119.

[28] R. Gençay, F. Selçuk, Extreme value theory and value-at-risk: relative performance in emerging markets, Int. J. Forecast. (2003) forthcoming.

[29] E. Ghysels, On the economics and econometrics of seasonality, in: C.A. Sims (Ed.), Sixth World Congress, Advances in Econometrics, Vol. 2, Cambridge University Press, Cambridge, 1994.

[30] R. Gençay, F. Selçuk, B. Whitcher, Differentiating intraday seasonalities through wavelet multi-scaling, Physica A (289) (2001a) 543-556.

[31] B. Efron, Bootstrap methods: another look at the jackknife, Ann. Stat. (7) (1979) 1-26.

[32] B. Gutenberg, C.F. Richter, Earthquake magnitude, intensity, energy and accelaration, Seismol. Soc. Am. Bull. (46) (1956) 105-145.

[33] F. Omori, On the after-shocks of earthquakes, J. Coll. Sci. Imp. Univ. Tokyo (7) (1894) 111-200. 